\title{
Dynamics in $\mathrm{Cu}_{46} \mathrm{Zr}_{42} \mathrm{Al}_{7} \mathrm{Y}_{5}$ melts: Interplay between packing density and viscosity
}

\author{
S. M. Chathoth, ${ }^{1, a)}$ B. Damaschke, ${ }^{1}$ J. P. Embs, ${ }^{2}$ and K. Samwer ${ }^{1}$ \\ ${ }^{1}$ I. Physikalisches Institut, Georg-August-Universität, 37077 Göttingen, Germany \\ ${ }^{2}$ Laboratory for Neutron Scattering, ETH Zurich, Paul Scherrer Institut, CH-5232 Villigen, \\ Switzerland and Physical Chemistry, Saarland University, D-66123 Saarbrcken, Germany
}

(Received 3 April 2009; accepted 2 May 2009; published online 22 May 2009)

\begin{abstract}
We have investigated microscopic dynamics in the bulk glass-forming $\mathrm{Cu}_{46} \mathrm{Zr}_{42} \mathrm{Al}_{7} \mathrm{Y}_{5}$ melts using quasielastic neutron scattering (QENS). Self-correlation functions show a fast $\beta$ and a slow $\alpha$-relaxation process. $\alpha$ relaxation exhibits stretching and is independent of momentum transfer and temperature. The melt is characterized by low packing density and high viscosity. The dynamics observed and the critical packing density derived from the QENS data are in good agreement with mode-coupling theory predictions for hard-sphere liquids. However, we can show that viscosity dominates over packing density in determining the atomic dynamics and glass-forming ability of the metallic melt. (C) 2009 American Institute of Physics. [DOI: 10.1063/1.3142389]
\end{abstract}

Even though there is no direct correlation between density $(\rho)$ and viscosity $(\eta)$ of liquids, the glass-forming melts are found to be highly dense and viscous. For example, the $\eta$ of the best glass-forming metallic liquids like $\mathrm{Pd}_{43} \mathrm{Ni}_{10} \mathrm{Cu}_{27} \mathrm{P}_{20} \quad$ (Ref. 1) (PdNiCuP) and $\mathrm{Zr}_{41.2} \mathrm{Ti}_{13.8} \mathrm{Ni}_{10} \mathrm{Cu}_{12.5} \mathrm{Be}_{22.5}$ (Ref. 2) is about three to four times larger than that of simple metallic and binary alloy liquids at their melting temperatures ${ }^{3}\left(T_{m}\right)$. The $\rho$ of metallic liquids can be better represented by packing density ${ }^{4}(\varphi)$, which is about $25 \%-35 \%$ higher than that of simple liquid metals. ${ }^{3,4}$ If we compare the $\eta$ and $\varphi$ of $\mathrm{Cu}_{46} \mathrm{Zr}_{42} \mathrm{Al}_{7} \mathrm{Y}_{5}$ (Ref. 5) and $\mathrm{Ni}_{60} \mathrm{Nb}_{34.8} \mathrm{Sn}_{5.2}$ melts, ${ }^{4}$ the $\eta$ of the former is greater than that of the latter but the $\varphi$ is larger for $\mathrm{Ni}_{60} \mathrm{Nb}_{34.8} \mathrm{Sn}_{5.2}$. Both melts are glass forming but the glass-forming ability (GFA) is different. ${ }^{6,7}$ We have studied the microscopic dynamics in $\mathrm{Ni}_{60} \mathrm{Nb}_{34.8} \mathrm{Sn}_{5.2}$ previously by QENS. ${ }^{4}$ In this letter, we report on our research in to bulk glass-forming $\mathrm{Cu}_{46} \mathrm{Zr}_{42} \mathrm{Al}_{7} \mathrm{Y}_{5}$ melts using QENS to understand the influence of the interplay between $\varphi$ and $\eta$ in microscopic dynamics and GFA of metallic melts.

Details of experiments as described in Ref. 8. Only $\mathrm{Cu}$ atoms possess incoherent scattering cross section in this alloy. So the resulting self-correlation functions $\Phi(q, t)$ represent the mean dynamics of $\mathrm{Cu}$ atoms in the melt. The sample was measured at room temperature to obtain the instrument energy resolution function. In the melt, the sample was measured at $1130 \mathrm{~K}$, which was close to the $T_{m}$ and at four more temperatures up to $1330 \mathrm{~K}$ with a step interval of $50 \mathrm{~K}$. The data were collected over a period of $4-5 \mathrm{~h}$ at each temperature. The scattering law, $S(q, \omega)$, was determined by normalizing the raw data to a vanadium standard, correcting for self-absorption and container scattering, interpolating to constant $q$, and symmetrizing with respect to energy with the detailed balance factor. Fourier deconvolution of $S(q, \omega)$ and normalization to 1 for $t=0$ gives the $\Phi(q, t)$. Figure 1 displays the $\Phi(q, t)$ of the liquid $\mathrm{Cu}_{46} \mathrm{Zr}_{42} \mathrm{Al}_{7} \mathrm{Y}_{5}$ at $q$ $=1.09 \AA^{-1}$, which clearly shows a two-step relaxation process. Between 0 and $0.3 \mathrm{ps}$, phonon leads to a rapid decay of

${ }^{a)}$ Electronic mail: schatho@gwdg.de.
$\Phi(q, t)$. As time increases, $\Phi(q, t)$ shows almost a constant value or a plateau for $f_{q}$. On approaching this plateau, before $\Phi(q, t)$ decays toward zero, a fast relaxation is clearly visible. Since the vibrational part of the spectrum [represented by the first data point in $\Phi(q, t)]$ is well separated from the relaxational parts, this gives an advantage for investigating the picosecond dynamics in the vicinity of $f_{q}$, the fast $\beta$-relaxation process. According to mode-coupling theory $(\mathrm{MCT})$, the time correlation function of the dynamical variable in the $\beta$-relaxation regime is expected to follow ${ }^{9}$ as

$$
\Phi_{q}(t)=f_{q}+h_{q} g_{\lambda}\left(t / t_{\sigma}\right),
$$

where the time dependent term $g_{\lambda}\left(t / t_{\sigma}\right)$ is completely determined by a single unique exponent parameter $\lambda$. Here, $f_{q}$ is the Debye-Waller factor, $h_{q}$ is the amplitude of $\beta$ relaxation, and $t_{\sigma}$ is the crossover time. The theory predicts the temperature variation of $t_{\sigma}$ and $h_{q}$ with the asymptotic scaling functions

$$
h_{q} \propto\left(T-T_{c}\right)^{1 / 2} \quad \text { and } \quad t_{\sigma} \propto\left(T-T_{c}\right)^{-1 / 2 a},
$$

where the exponent $a$ is determined by $\lambda$. The values of $a$, $g_{\lambda}$, and $\gamma$ as a function of $\lambda$ were obtained from Ref. 9. The

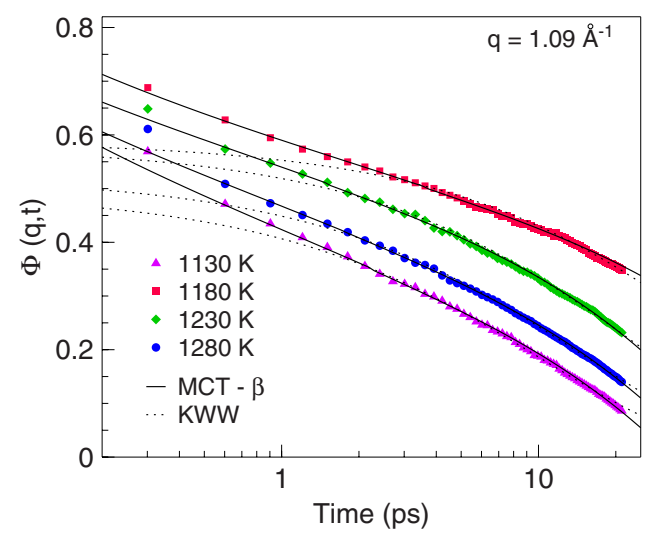

FIG. 1. (Color online) The $\Phi(q, t)$ of $\mathrm{Cu}_{46} \mathrm{Zr}_{42} \mathrm{Al}_{7} \mathrm{Y}_{5}$ melts obtained on the neutron time-of-flight spectrometer, FOCUS. The solid lines are from fits using the MCT $\beta$-scaling law [Eq. (1)] with $\lambda=0.77$. The dotted lines are fits with the KWW function with $\beta_{q}=0.7$ in the $\alpha$-relaxation regime. 


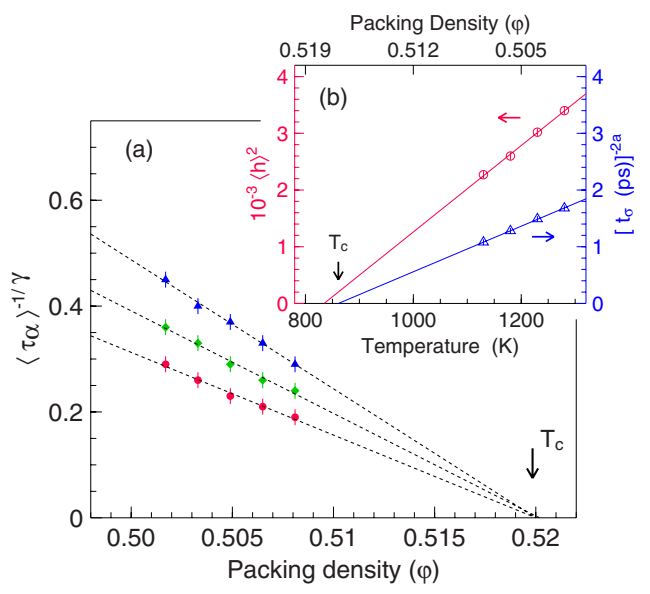

FIG. 2. (Color online) (a) The $\varphi$ depends on the mean relaxation time of the $\alpha$-process rescaled using Eq. (3). The dotted lines are the linear fit crossing at zero, $\varphi_{c}=0.519 \pm 0.002$. (b) The temperature dependence of the amplitude $\left\langle h_{q}\right\rangle$ and time scale $\left\langle t_{\sigma}\right\rangle$ of the $\beta$-relaxation process according to Eq. (2). Both lines are extrapolated consistently to obtain the MCT $T_{c}$ at $840 \pm 5 \mathrm{~K}$. The corresponding $\varphi_{c}$ is $0.517 \pm 0.002$.

two-step decay can be fitted with an MCT $\beta$-scaling law [Eq. (1)] in the range from 0.3 to $15 \mathrm{ps}$. Details of fitting iteration are described in Ref. 8. The temperature dependence of the amplitude $h_{q}$ and the crossover time $t_{\sigma}$ strongly indicate the slowing down of the relaxational dynamics upon cooling.

Figure 2(b) displays the temperature variation of $h_{q}$ and $t_{\sigma}$ for the fast $\beta$ relaxation in liquid $\mathrm{Cu}_{46} \mathrm{Zr}_{42} \mathrm{Al}_{7} \mathrm{Y}_{5}$, given according to Eq. (2). The temperature dependence of $h_{q}$ and $t_{\sigma}$ is in accordance with the MCT predictions with an exponent $a=0.295$, based on $\lambda=0.77$. When $h_{q}$ and $t_{\sigma}$ are extrapolated $T_{\text {liq }}$, a consistent value for the MCT glasstransition temperature, $T_{c}=840 \pm 10 \mathrm{~K}$ is obtained. In the fluid regime, the $T_{c}$ derived from idealized MCT shows its universal character, i.e., $T_{c} \simeq 1.2 T_{g}$. The corresponding $\varphi$ of $\mathrm{Cu}_{46} \mathrm{Zr}_{42} \mathrm{Al}_{7} \mathrm{Y}_{5}$ melt at $840 \pm 10 \mathrm{~K}$ is $0.517 \pm 0.002$. The $\Phi(q, t)$ in the $\alpha$-relaxation time was fitted with Kohlrausch-Williams-Watts (KWW) function, $\Phi(q, t)$ $=f_{q} \exp \left[\left(-t / \tau_{q}\right)^{\beta_{q}}\right]$ (Fig. 1). The data points were initially fitted using an arbitrary value for $\beta_{q}$. Over the assessable $q$ range, $\beta_{q}$ is found to vary around a value of $0.70 \pm 0.04$. Further analysis was carried out by fixing the $\beta_{q}$ value to a constant, 0.70 . This fitting procedure shows that the data measured at different temperatures can be best described by a temperature and a $q$ independent stretching exponent $\left(\beta_{q}\right)$. MCT gives a microscopic explanation for stretching in terms of a nonlinear coupling of density fluctuation caused by a feedback effect in the dense liquids. On the other hand, $\eta$ is characterized by resistance to move the atoms. So at high $\eta$ and comparatively low $\varphi$, the feedback effect is negligible as compared to the viscous resistance. This in fact stabilizes the stretching of $\Phi(q, t)$ over wide temperature range. In contrast, $\mathrm{Ni}_{60} \mathrm{Nb}_{34.8} \mathrm{Sn}_{5.2}$ and $\mathrm{Ni}_{59.5} \mathrm{Nb}_{40.5}$ melt shows a temperature-dependent stretching. This is due to low $\eta$ and high $\varphi$, and the $\varphi$ changes with temperature which in turn reduces the feedback, as a consequence changing the $\beta_{q}$.

The resulting mean relaxation time obtained as $\left\langle\tau_{q}\right\rangle$ $=\int_{0}^{\infty} \Phi(q, t) / f_{q}=\tau_{q} \beta_{q}^{-1} \Gamma\left(\beta_{q}^{-1}\right)$, where $\Gamma$ is the gamma function. In glass-forming liquids, $\left\langle\tau_{\alpha}\right\rangle$ of the collective motion of atoms are strongly influenced by the $\varphi .^{10}$ The MCT predicts the variation of $\left\langle\tau_{\alpha}\right\rangle$ with $\varphi$ as

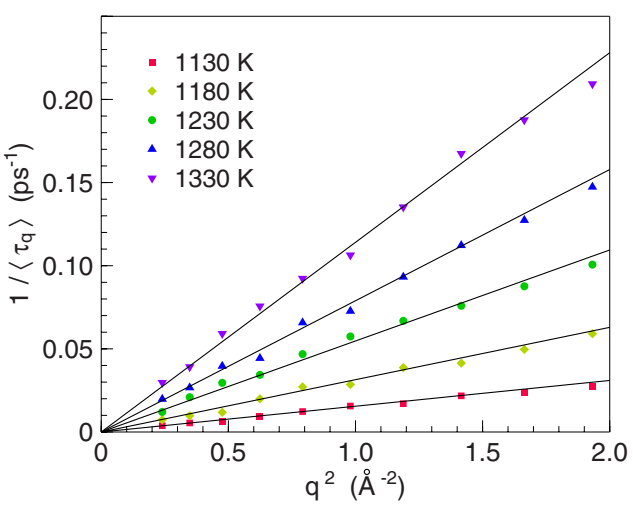

FIG. 3. (Color online) $q^{2}$ depends on the inverse of $\left\langle\tau_{q}\right\rangle$ observed for five different temperatures. At $q$ values, $q \ll q_{0}$, which shows a linear dependence. The straight line is the linear fit and the slope gives the $D$.

$$
\left\langle\tau_{\alpha}\right\rangle^{-1 / \gamma} \propto\left[\varphi-\varphi_{c}\right] .
$$

Figure 2(a) displays $\left\langle\tau_{\alpha}\right\rangle^{-1 / \gamma}$ as a function of the $\varphi$ with $\gamma$ $=2.7$ for the three different values of $q$. The variation of $\left\langle\tau_{\alpha}\right\rangle^{-1 / \gamma}$ with $\varphi$ is linear, as predicted by the MCT. Linear fits to the data points cross at zero, where the viscous flow arrest and hopping process become the dominant transport mechanism or the $\varphi_{c}$. The values of $\varphi_{c}$ obtained from the above analysis was $0.519 \pm 0.001$.

Additionally, $\left\langle\tau_{\alpha}\right\rangle$ is proportional to $1 / q^{2}$ as expected in the hydrodynamic limit for $q \ll q_{0}{ }^{11}$ (Fig. 3). This enables us to determine the average self-diffusivity $D=\frac{1}{q^{2}\left\langle\tau_{q}\right\rangle}$. The $D$ obtained at $1130 \mathrm{~K}$ is $1.82 \pm 0.16 \times 10^{-10} \mathrm{~m}^{2} \mathrm{~s}^{-1}$ and at the highest measured temperature, i.e., at $1330 \mathrm{~K}, D$ obtained was $9.60 \pm 0.16 \times 10^{-10} \mathrm{~m}^{2} \mathrm{~s}^{-1}$. This is comparatively larger than the better metallic glass-forming $\mathrm{Pd}_{43} \mathrm{Ni}_{10} \mathrm{Cu}_{27} \mathrm{P}_{20}$ $\left(D=3 \pm 1 \times 10^{-11} \mathrm{~m}^{2} \mathrm{~s}^{-1}\right),{ }^{12} \mathrm{Zr}_{41.2} \mathrm{Ti}_{13.8} \mathrm{Cu}_{12.5} \mathrm{Ni}_{10} \mathrm{Be}_{22.5}$, and $\mathrm{Zr}_{46.8} \mathrm{Ti}_{8.2} \mathrm{Cu}_{7.5} \mathrm{Ni}_{10} \mathrm{Be}_{27.5}$ at their liquidus temperatures. ${ }^{13}$ The temperature dependence of the $D$ is well described by a $\tau$ scaling law of MCT, $D \propto\left[\left(T-T_{c}\right) / T_{c}\right]^{\gamma}$. In Fig. 4, the solid line is the $\tau$-scaling law fit to the measured $D$ with $T_{c}$ $=900 \mathrm{~K}$ and $\gamma=2.7$. The $\varphi_{c}$ at $900 \mathrm{~K}$ is $0.516 \pm 0.001$.

The average value for $\varphi_{c}$ obtained from $\beta$ relaxation, $\left\langle\tau_{\alpha}\right\rangle$ of the $\alpha$ process, and $\tau$-scaling analysis is $0.517 \pm 0.003$. This value is about $6.3 \%$ lower than that observed in $\mathrm{Ni}_{60} \mathrm{Nb}_{34.8} \mathrm{Sn}_{5.2}$ and $\mathrm{Ni}_{59.5} \mathrm{Nb}_{40.5}$ liquids. However it is close to the MCT calculation for monoatomic hard-sphere liquids. The MCT calculation shows that the $\varphi_{c}$ for dynamic cross

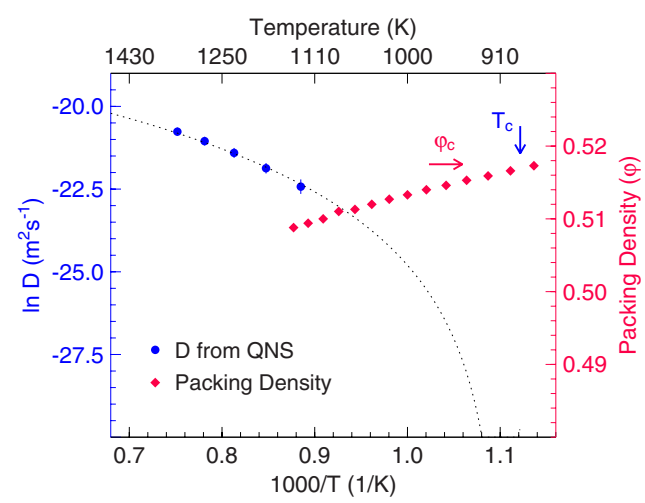

FIG. 4. (Color online) The $D$ of $\mathrm{Cu}$ atoms in the liquid $\mathrm{Cu}_{46} \mathrm{Zr}_{42} \mathrm{Al}_{7} \mathrm{Y}_{5}$ as observed by QENS. The dotted curve is the $\tau$-scaling fit to $D$ with $T_{0}$ $=900 \mathrm{~K}$ and $\gamma=2.7$. The corresponding $\varphi_{c}$ is $0.516 \pm 0.002$. 
over is 0.515 using the Percus-Yevick structure factor as an input. ${ }^{10}$ Rather $S(q)$ obtained from simulation as input gives $\varphi_{c}=0.525$. $^{10}$ A binary mixture with size ratio of 0.83 and using $S(q)$ from the simulation as input gives $\varphi_{c}=0.545$. $^{10}$ Theoretical studies indicate a variation of $\varphi_{c}$ using $S(q)$ obtained in different ways as an input and mixing of atoms with different size ratio. Generally it is observed that $T_{c}$ $\simeq 1.2 T_{g}$ and this is valid in many polymeric, ${ }^{14}$ organic, ${ }^{15}$ and metallic ${ }^{12}$ glass-forming liquids. The bulk-glass-forming $\mathrm{Cu}_{46} \mathrm{Zr}_{42} \mathrm{Al}_{7} \mathrm{Y}_{5}$ melts give similar result but the packing density at $1.2 T_{g}$ is only 0.519 . In this liquid, the major contribution to the slowing down of atomic motion with decrease in temperature is due to the increase in $\eta$ but not by increase in $\varphi$. Due to its high $\eta$, the liquid becomes frozen before it reaches the maximum possible $\varphi$. This must be the reason for the comparatively low value of $\varphi_{c}$. Nevertheless, the $\varphi_{c}$ derived from the QNS study is within the MCT results for a hard-sphere liquid.

The GFA of metallic glass forming melts can be characterized by their critical-casing thickness. On comparing the GFA of $\mathrm{Cu}_{46} \mathrm{Zr}_{42} \mathrm{Al}_{7} \mathrm{Y}_{5}$ and $\mathrm{Ni}_{60} \mathrm{Nb}_{34.8} \mathrm{Sn}_{5.2}$, the former can mold-cast more than $10 \mathrm{~mm}$ but $\mathrm{Ni}_{60} \mathrm{Nb}_{34.8} \mathrm{Sn}_{5.2}$ can only cast up to $2 \mathrm{~mm}$ diameter rods. $\mathrm{Ni}_{60} \mathrm{Nb}_{34.8} \mathrm{Sn}_{5.2}$ melt is the highest packed metallic liquid ever known but the high value of $\varphi$ did not contribute to its GFA. Similarly, if we compare the $\varphi$ of $\mathrm{Ni}_{80} \mathrm{P}_{20}, \mathrm{Pd}_{40} \mathrm{Ni}_{40} \mathrm{P}_{20}$, and $\mathrm{PdNiCuP}$, it is similar at their $T_{m}{ }^{16}$ However the viscosities of these liquids are very different at their $T_{m} \cdot{ }^{1,17} \mathrm{PdNiCuP}$ can mold cast more than 10 $\mathrm{cm}$ rods but $\mathrm{Ni}_{80} \mathrm{P}_{20}$ can make amorphous only in ribbons. Thus, $\eta$ is the determining factor of GFA rather than the $\varphi$ of metallic liquid.

In summary, we used QENS to investigate microscopic dynamics in $\mathrm{Cu}_{46} \mathrm{Zr}_{42} \mathrm{Al}_{7} \mathrm{Y}_{5}$ melts. The $\Phi(q, t)$ show fast $\beta$ and slow $\alpha$ relaxation. $\alpha$ relaxation shows stretching and the $\beta_{q}$ value is independent of $q$ and temperature. We believe that stretching of the $\Phi(q, t)$ is stabilized due to the high $\eta$ and comparatively low $\varphi$ of the melts. The $\varphi_{c}$ obtained from $\beta$-scaling analysis, $\left\langle\tau_{q}\right\rangle$ of $\alpha$-process, and $\tau$-scaling analysis of $D$ is $0.517 \pm 0.003$. This value is comparatively smaller than that observed in other glass-forming metallic melts. The reason for this is believed to be the high viscosity, which dominates over packing density of the melt in determining the atomic dynamics. The value of $\varphi_{c}$ obtained for $\mathrm{Cu}_{46} \mathrm{Zr}_{42} \mathrm{Al}_{7} \mathrm{Y}_{5}$ melt is within the range of the MCT prediction for hard-sphere liquids.

We thank PSI for the beam time and the EU-NMI3 program (FP6) for funding. Additional support provided by the DLR through Grant No. 50WM0541, DFG SFB 602, and the Leibniz program are gratefully acknowledged.

${ }^{1}$ P. H. Haumesser, J. P. Garandet, J. Bancillon, M. Daniel, I. Campbell, and P. Jackson, Int. J. Thermophys. 23, 1217 (2002)

${ }^{2}$ A. Masuhr, T. A. Waniuk, R. Busch, and W. L. Johnson, Phys. Rev. Lett. 82, 2290 (1999).

${ }^{3} \mathrm{U}$. Balucani and M. Zoopi, Dynamics in Liquid State (Clarendon, Oxford, 1994).

${ }^{4}$ S. M. Chathoth, D. Damaschke, M. M. Koza, and K. Samwer, Phys. Rev. Lett. 101, 037801 (2008).

${ }^{5}$ G. J. Fan, J. J. Z. Li, W.-K. Rhim, D. C. Qiao, H. Choo, and P. K. Liaw, Appl. Phys. Lett. 88, 221909 (2006).

${ }^{6}$ S. Mukherjee, J. Schroers, Z. Zhou, W. L. Johnson, and W.-K. Rhim, Acta Mater. 52, 3689 (2004).

${ }^{7}$ D. Xu, G. Duan, and W. L. Johnson, Phys. Rev. Lett. 92, 245504 (2004).

${ }^{8}$ S. M. Chathoth and A. Podlesnyak, J. Appl. Phys. 103, 013509 (2008).

${ }^{9}$ W. Götze, J. Phys.: Condens. Matter 2, 8485 (1990).

${ }^{10}$ G. Foffi, W. Götze, F. Sciortino, P. Tartaglia, and Th. Voigtmann, Phys. Rev. E 69, 011505 (2004).

${ }^{11}$ J. P. Boon and S. Yip, Molecular Hydrodynamic (McGraw-Hill, New York, 1980).

${ }^{12}$ A. Meyer, Phys. Rev. B 66, 134205 (2002).

${ }^{13}$ A. Meyer, W. Petry, M. Koza, and M.-P. Macht, Appl. Phys. Lett. 83, 3894 (2003).

${ }^{14}$ G. Floudas, W. Steffen, L. Giebel, and G. Fytas, Prog. Colloid Polym. Sci. 91, 28 (1993).

${ }^{15}$ J. Wuttke, J. Hernandez, G. Li, G. Coddens, H. Z. Cummins, F. Fujara, W. Petry, and H. Sillescu, Phys. Rev. Lett. 72, 3052 (1994).

${ }^{16}$ S. M. Chathoth, A. Meyer, M. M. Koza, and F. Juranyi, Appl. Phys. Lett. 85, 4881 (2004).

${ }^{17}$ Y. Nishi and A. Yoshihiro, Scr. Metall. 19, 1023 (1985). 